\title{
Zygomatico-Maxillary Complex Fracture Fixation: How Much Is Enough?
}

\author{
Mohit Jain $^{1,2,3 *}$, Geetanjali Mandlik ${ }^{1}$ and Mukul Padhye ${ }^{1}$ \\ ${ }^{1}$ Department of Oral and Maxillofacial Surgery, D Y Patil University, India \\ ${ }^{2}$ Bhagwan Mahaveer Hospital, India \\ ${ }^{3}$ Inlaks General Hospital, India \\ *Corresponding Author: Mohit Jain, Department of Oral and Maxillofacial Surgery, D Y Patil University, India.
}

Received: November 01, 2019; Published: November 21, 2019

DOI: $10.31080 /$ ASDS.2019.03.0706

\begin{abstract}
The prominence of the zygomatic region predisposes it to bearing the brunt of facial injuries. Due to the architectural pattern of the mid-face, the zygoma generally fractures at four of its articulations (tetrapod fracture) making displacement possible in multiple planes and directions. Ocular injuries, occlusal disharmony and cosmetic deformities are sequelae of zygomatic complex fractures. Due to multiple fracture points, the number of fixation points for adequate reduction and optimal fixation is a point of discussion in the literature. As on date, there is no specific protocol or any common consensus on the fixation pattern in a tetrapod zygomaticomaxillary complex fracture. Several studies claim that one or two-point fixation is enough but these studies are not backed by long term follow-ups, nor do any of these follow a specific treatment protocol. We follow a combined approach by Ed Ellis., et al. and the AOCMF guidelines and generally prefer a three or four-point fixation depending on the comminution and displacement for tetrapod zygomatico-maxillary complex fractures. The case reported is a typical tetrapod fracture and was planned for a three-point fixation along with closed reduction of the zygomatic arch using the Keens approach. The graduated meticulous approach of alignment of the zygoma with the greater wing of sphenoid, adequate reduction and fixation at the fractured points with reconstruction of the buttresses will give optimal results which in turn will help in maintaining facial height, facial width and restore form, function and esthetics.
\end{abstract}

Keywords: Zygomatic Fractures; Zygomatico-Maxillary Complex Fractures; Fixation Points

\section{Abbreviations}

F-Z Suture: Fronto-Zygomatic Suture; ZM Buttress: ZygomaticoMaxillary Buttress; IOR: Infra-Orbital Rim; Z-S: ZygomaticoSphenoid Suture; TZMC: Tetrapod Zygomatico-Maxillary Complex; ZMC: Zygomatico-Maxillary Complex

\section{Introduction}

The zygoma is an enigmatic facial bone with its confluence influencing a large portion of the facial functional and cosmetic features. It's prominence makes it vulnerable to bearing the brunt of facial injuries [1] and so it represents $13 \%$ of all maxillofacial injuries and is the second most common bone fractured following the nasal bone [1,2]. The zygoma is most vulnerable to fracture beyond the dorsum of the nose. Due to its position, a fractured zygoma could lead to ophthalmic injuries, occlusal disturbances, cosmetic deformities or a combination of these. The fractured zygoma biomechanically has six possible directions of displacement i.e. rotations and translations around the $\mathrm{X}, \mathrm{Y}$ and $\mathrm{Z}$ axis.

The buttresses of the face need to be considered while working on the zygomatic complex as one of the purposes of fixation is to reconstruct these buttresses. The medial or nasomaxillary buttress, the pterygomaxillary or posterior buttress, which connects the maxilla posteriorly to the sphenoid bone and the 
lateral or zygomaticomaxillary (ZM) buttress come into play in zygomatico-maxillary complex (ZMC) fractures. The ZM buttress is important as it connects the lateral maxillary alveolus to the zygomatic process of the temporal bone. These buttresses give structural stability to the facial skeleton to withstand forces. The ZMC fracture can impair ocular and masticatory function as well as cause psychosocial problems. Inaccurately reduced or fixed fractured zygomas can lead to suboptimal projection of the malar contour leading to facial asymmetry. Inadequate fixation can also cause instability or movements in the complex even after fixation due to masticatory forces. Salvage surgery to reconstruct the deformities and correct asymmetry is always a surgical challenge.

\section{The Tetrapod ZMC (TZMC) fracture}

Due to the architectural form of the zygomatic bone, it generally can withstand great forces of trauma without fracturing, though inadvertently it tends to fractures at its articulations [3]. Contrary to the previously believed idea of the zygomatic complex fracture as a tripod fracture, the currently perceived notion is that it is a tetrapod fracture [4].

The zygoma can fracture at four of its articulations viz., the zygomatico-frontal suture (F-Z), the zygomaticomaxillary buttress (ZMB), the zygomatico-temporal suture (Z-T) and the zygomaticosphenoid suture (Z-S) [5]. Displacement and fractures can occur at one, two, three or all four articulations depending upon the velocity and direction of the force inducing trauma, making it a point of discussion in the literature. Accurate reduction at the Z-S suture can be a reliable sign of an accurately reduced zygomatic complex especially in laterally displaced fractures [3].

The clinical picture

A typical zygomatic complex fracture will show ocular signs and cosmetic changes frequently, and occlusal disturbances infrequently. Ocular signs range from corneal irritations to major complications such as hemorrhage and optic nerve injury. The frequency of injury to the eye ranges from 2.7 to $96 \%$ as these injuries destroy the orbital skeleton [6]. Periorbital edema and periorbital ecchymoses are a routine finding following trauma. Subconjunctival haemorrhage (SCH-86\%), hyphema (13\%), diplopia (20\%), corneal injury (32\%), enophthalmos are seen more frequently and seldom does the patient present with retrobulbar hemorrhage, decreased visual acuity and very rarely is optic nerve injury seen [7].
Depression of the malar contour is frequently seen in such fractures, leading to a cosmetic deformity. Impingement of the zygomatic arch on the coronoid can lead to occlusal disturbances. Inferior displacement of the maxilla due to fracture of the ZM buttress is also a regular finding causing occlusal disharmony.

\section{Discussion}

\section{How much is Enough?}

Displacement and rotation are the most common indication for surgery in TZMC fractures. Three-dimensional displacement can occur in such fractures. Currently, there is no specific algorithm or treatment protocol or a common consensus for fixation of ZMC fractures. Careful diagnosis, meticulous planning and adequately optimum treatment are the pillars of a successful ZMC fracture management, which optimally restores the form, function and esthetics of the ZMC.

Closed reduction is an option in isolated, minimally displaced zygoma fractures but not in a tetrapod complex as displaced TZMC fractures are vulnerable to secondary malposition as a result of masticatory forces even after some kind of fixation [3]. Displacement in the zygoma after reduction can result in delayed development of malar asymmetry and vertical dystopia.

A survey answered by over 1600 ENT, OMFS and plastic surgeons showed 81\% would choose ORIF for ZMC fractures [8]. Hwang., et al. [9] and Nezhad [10] insisted that one-point fixation was enough to stabilise a displaced ZMC fracture. Choi., et al. [11] used 2-point fixation for accurate correction. Fujioka., et al. [12] stated that on-point fixation at the ZM buttress is enough to provide good stability, while Haider., et al. [13] found two-point fixation more stable. These studies may be considering the incision scar as a factor for not doing three-point fixation, although they do not report whether their cases were tetrapod or tripod fractures. Olate., et al. recommended fixation of the infra-orbital rim (IOR) and the F-Z suture for a displaced ZMC fracture, and for cases with displacement greater than $5 \mathrm{~mm}$, use of 3-point fixation was deemed suitable [14].

Translatory movement and rotation can be controlled by a miniplate at the F-Z suture, which will resist forces along an axis perpendicular to the plane of the plate. But this fixation does not resist forces along the linear plane of the plate. A favorable situation can be created by choosing three fixation points that are 
not collinear. Pearl [15], reported that it is essential to reposition the zygoma at a minimum of three locations to achieve correction in three dimensions. He also stated that inspite of reduction at the F-Z suture and the IOR, lateral rotation can persist in the region of the anterior ZM buttress leading to intra-orbital volume expansion behind the globe. Several studies [16,17] have analyzed miniplate fixation for stabilizing fractured zygoma in human skulls. They reported that three-point fixation at the ZM buttress, the F-Z suture and the infra-orbital rim conferred maximum stability against forces matching physiological stresses.

Literature states that most of the surgeons carry out orbital floor exploration in cases of primary diplopia or evidence of a comminuted TZMC fractures only [18]. Baylan., et al. conducted a survey where they reported that $35 \%$ of facial surgeons would carry out an orbital floor exploration in all displaced ZMC fracture cases [8]. We prefer to carry out orbital floor exploration only if there is evidence of primary diplopia, enophthalmos more than 5 mm or an entrapped ocular muscle.

Although there is no specific protocol for the number of fixation points for a ZMC fracture, we consider the following two proposed algorithms: The Ed Ellis Algorithm (for isolated displaced ZM fractures) [19] and the AOCMF guideline (for TZMC fractures). According to the Ed Ellis Algorithm, the need for internal orbital reconstruction is to be evaluated first. The limitation of this algorithm is that it does not take fracture of the infra-orbital rim into consideration if internal orbital reconstruction is not required. Also, it does not take into account TZMC fractures. The AO principle for a TZMC fracture suggests exposing all fractures but plating the fracture at the F-Z suture first with a 4-hole with gap plate with just 2 screws initially, with the remaining two screws to be placed at the end after all fractures have been fixed.

Selection of fixation points are determined by the influence of the points on optimum reduction and the displacement of the fractures. One-point fixation could be optimum for isolated displaced ZM buttress fractures with undisplaced or no fractures at the F-Z and the IOR. When doing a two-point fixation, the ZM buttress invariably needs to be fixed as it requires the greatest stability, with the F-Z or IOR fixation depending on the fracture site and displacement. Seldom would you encounter an isolated displaced infra-orbital rim fracture or an isolated displaced F-Z fracture which needs fixation. In most cases they are always accompanied by either the arch fracture or a ZM buttress fracture.
Three-point fixation is generally planned for the FZ, the ZM buttress and the IOR. In such cases we follow the AO algorithm, placing 2 screws at the F-Z first. In cases of a concomitant arch fracture, which can be reduced intraorally (Keens approach), threepoint fixation suffices. A 4-point fixation, where the fourth point of fixation is the fractured zygomatic arch, is planned only for a comminuted TZMC fracture with a cosmetic deformity. In such cases, the exposed zygomatic arch may be a good reference point to check if the AP dimension of the mid-face has been restored. Whenever possible, it is advisable to get adequately optimal reduction at the Z-S suture and the lateral orbital wall as that generally indicates an almost perfect reduction of the zygoma in all planes. We use the tried and tested approaches for exposing the fractures. The lateral eyebrow approach for the F-Z, the stepped subciliary approach or the transconjunctival approach for the IOR, the intra-oral vestibular incision for the ZM buttress and the hemicoronal approach in cases of 4-point fixation in comminuted TZMC fractures.

Although in a TZMC fracture we prefer a four-point fixation, there are certain disadvantages to it. More invasive approaches, expert surgical skill requirement, longer operative time, implanting more hardware in the skeleton and increased cost of surgery are certain factors to be taken into consideration. Surgical approaches and their complications can only be compared when outcome measurements are objectively assessed, which follows protocol management and long-term follow up [3]. The preference for open reduction and internal fixation of zygomatic fractures at three or four points has continued to grow in response to observations of inadequate results from two-point and one-point fixation techniques. The graduated meticulous approach of alignment of the zygoma with the greater wing of sphenoid, adequate reduction and fixation at the 3 or 4 points with reconstruction of the buttresses will give optimal results which in turn will help in maintaining facial height, facial width and restore form, function and esthetics.

\section{Case Report}

The patient $28 / \mathrm{F}$ presented with an alleged history of a road traffic accident leading to maxillofacial trauma. Clinically she presented to our Centre on the day of trauma with left peri-orbital ecchymoses, left peri-orbital edema (Figure 1 and 2), left $\mathrm{SCH}$, depressed left malar contour (Figure 3) and a malocclusion with an open bite on the right side (Figure 4 and 5). 3DCT evaluation showed a displaced TZMC fracture (Figure 6 and 7). 


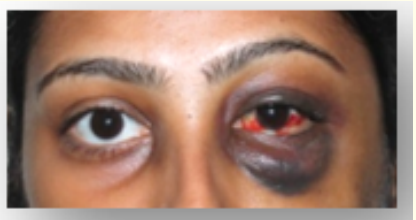

Figure 1: Left Peri-Orbital Edema, SCH And Ecchymoses.

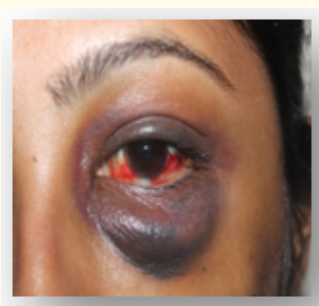

Figure 2: Left Peri-Orbital Edema, SCH and Ecchymoses.

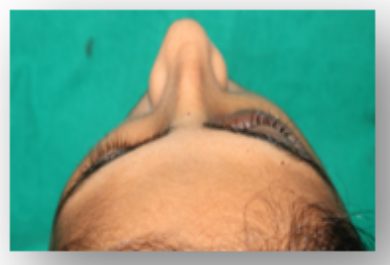

Figure 3: The Birds-Eye View - Depressed Left Malar Contour.

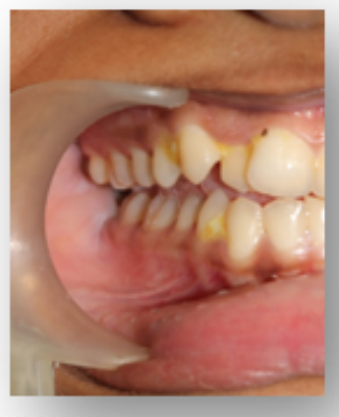

Figure 4: Malocclusion with a right side open bite.
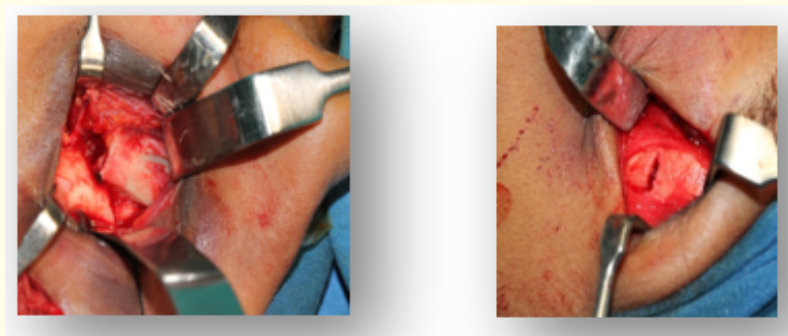

Figure 7: Exposed fracture sites.

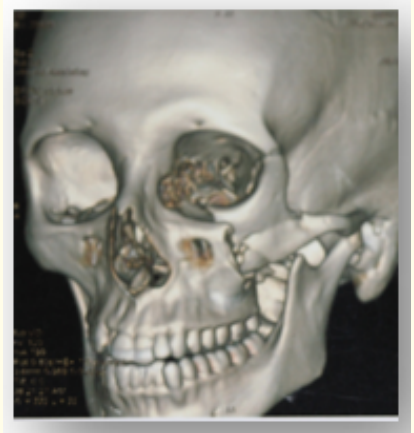

Figure 5: 3DCT showing a displaced TZMC fracture.

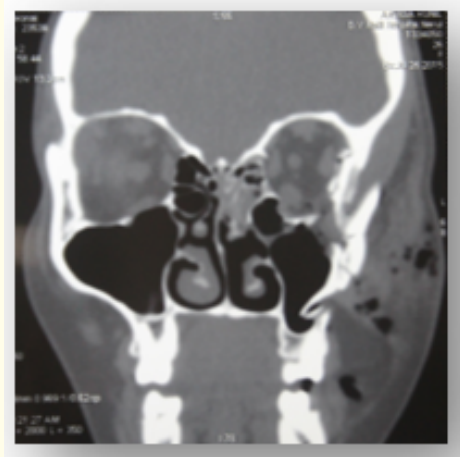

Figure 6: Coronal sections showing a displaced TZMC fracture.

A three-point fixation was planned with closed reduction of the zygomatic arch using the Keens approach. As there was no evidence of primary diplopia, enophthalmos or restriction in 
ocular muscle movement (Using the Forced Duction Test) in all 9 gazes, exploration of the orbital floor was not planned. Surgery was scheduled 5 days after the incident of trauma for the edema to subside. The F-Z suture was exposed first via a lateral eyebrow incision, followed by the ZM buttress which was exposed by an intra-oral vestibular incision. After checking for reduction, there was still some resistance in the movements of the complex. The IOR was then exposed via a stepped sub ciliary incision. Reduction was achieved, and the arch was also elevated and reduced at this stage by the Keens approach. Following optimal reduction and applying the AO protocol, 2 screws of the 4-hole plate were first placed at the F-Z suture followed by plating of the ZM buttress with a $2.0 \mathrm{~mm}$ L-plate, followed by the IOR fixation with a $1.5 \mathrm{~mm}$ 7-hole orbital plate. After confirming the proper repositioning of the zygoma, finally the last 2 screws were placed at the remaining 2 holes of the 4-hole plate at the F-Z suture. Adequate stabilization was achieved after fixation. Closure was performed using 3 - 0 vicryl for subcutaneous and muscle approximation and 4 - 0 prolene was used for skin closure. Post-operative recovery was uneventful and the patient was discharged on the 3rd post-operative day. Elevation and symmetry of the malar contour was achieved along with correction of the malocclusion and reconstructing the buttresses, thereby restoring form, function and esthetics.

Case pictures

Pre-operative

Extra-Oral Photographs

Intra-Oral Photograph

Ct Scans

Intra-Operative Photographs

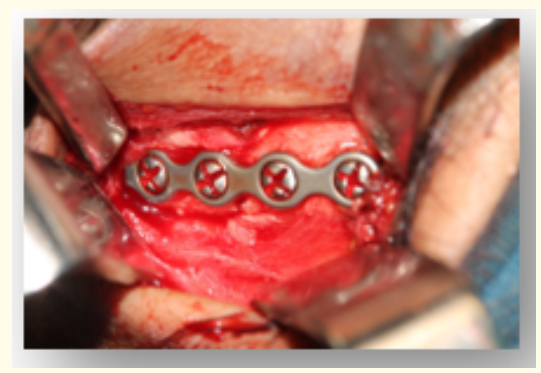

Figure 8: F-Z fracture fixation.

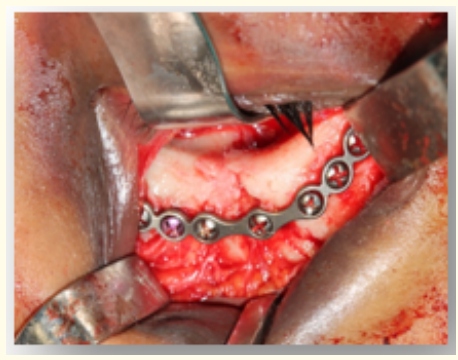

Figure 9: IOR fracture fixation.

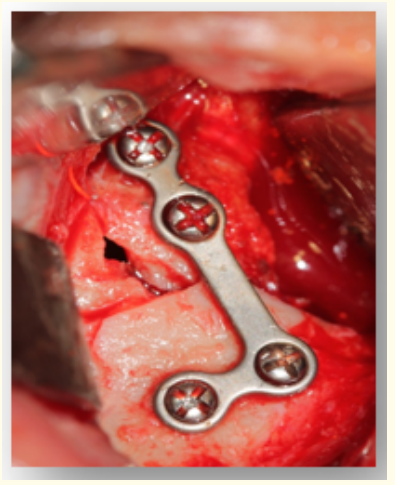

Figure 10: ZM butress fracture fixation.

\section{Post-Operative Photographs (14 Days)}

Extra-Oral + Intra-Oral Photographs

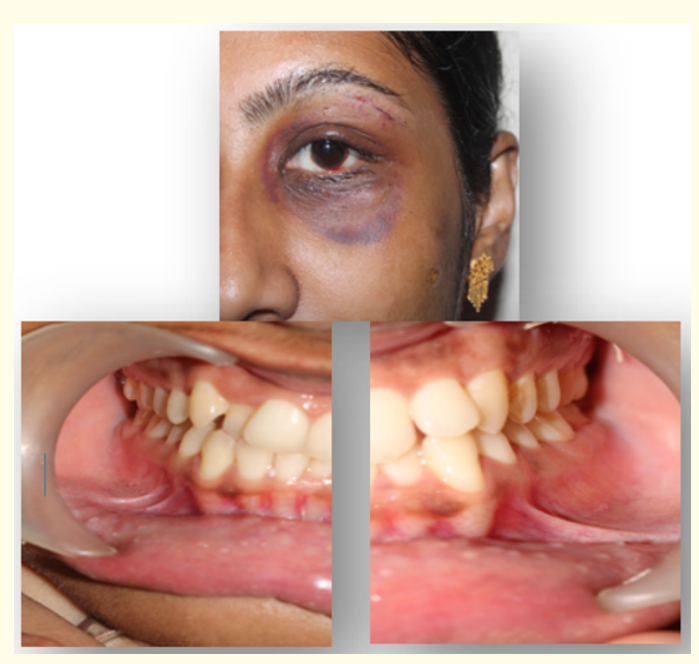

Figure 11: Uneventful healing with stable occlusion. 
Post-Operative Radiograph

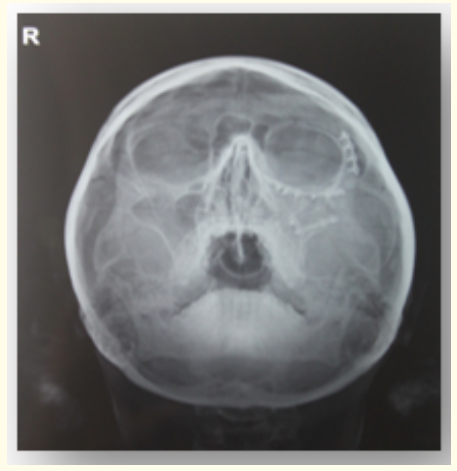

Figure 12: PA Waters view showing the 3-point fixation for the left ZMC.

\section{Bibliography}

1. Chowdhury., et al. "Etiology and Management of Zygomaticomaxillary Complex Fractures in the Armed Forces". Medical Journal, Armed Forces India 61.3 (2005): 238-240.

2. Tadj A and Kimble FW. "Fractured zygoma”. ANZ Journal of Surgery 73 (2003): 49-54.

3. Rana Majeed., et al. "Surgical treatment of zygomatic bone fracture using two points fixation versus three point fixation-a randomised prospective clinical trial". Trials 13(2012): 36.

4. Daniel Bradley., et al. "Kathleen Fan Surgical management of zygomatic complex fractures in a major trauma centre". Plastic and Aesthetic Research 6 (2019): 11.

5. Lee Edward I., et al. "Optimizing the surgical management of zygomaticomaxillary complex fractures". Seminars in Plastic Surgery 24.4 (2010): 389-397.

6. Al-Qurainy IA., et al. "A The characteristics of midfacial fractures and the association with ocular injury: a prospective study". British Journal of Oral and Maxillofacial Surgery 29.5 (1991): 291-301.

7. Malik Altaf Hussain., et al. "Ocular Injuries in Patients of Zygomatico-Complex (ZMC) Fractures". Journal of Oral and Maxillofacial Surgery 16.2 (2017): 243-247.

8. Baylan JM., et al. "Management of zygomatic fractures: a national survey". Journal of Craniofacial Surgery 27 (2016): 1571-1575.
9. Hwang KJ. "One-point fixation of tripod fractures of zygoma through a lateral brow incision". Journal of Craniofacial Surgery 21.4 (2010):1042-1044.

10. Mohammadinezhad C. "Evaluation of a single miniplate use in treatment of zygomatic bone fracture". Journal of Craniofacial Surgery 20.5 (2009): 1398-1402.

11. Choi KY., et al. "Feasibility of 4-point fixation using the preauricular approach in a zygomaticomaxillary complex fracture". Journal of Craniofacial Surgery 24.2 (2013): 557-562.

12. Fujioka M., et al. "Stability of one-plate fixation for zygomatic bone fracture". Plastic and Reconstructive Surgery 109 (2002): 817-818.

13. Haider Z. "Fractures of the zygomatic complex in South East Region of Scotland". British Journal of Oral and Maxillofacial Surgery 15 (1978): 265-267.

14. Olate S., et al. "Surgical approaches and fixation patterns in zygomatic complex fractures". Journal of Craniofacial Surgery 21 (2010):1213-1217.

15. Pearl RM. "Treatment of enophthalmos". Clinics in Plastic Surgery 19 (1992): 99-111.

16. Davidson J., et al. "Zygomatic fractures: comparison of methods of internal fixation". Plastic and Reconstructive Surgery 86 (1990): 25-32.

17. O'Hara DE., et al. "The role of microfixation in malar fractures: a quantitative biophysical study". Plastic and Reconstructive Surgery 97 (1996): 345-353.

18. Kovacs AF and Ghahremani M. "Minimization of zygomatic complex fracture treatment". International Journal of Oral and Maxillofacial Surgery 30 (2001): 380-383.

19. Ellis E. "Perez D An algorithm for the treatment of isolated zygomatico-orbital fractures". International Journal of Oral and Maxillofacial Surgery 72.10 (2014): 1975-1983.

\section{Volume 3 Issue 12 December 2019}

(C) All rights are reserved by Mohit Jain., et al. 